\title{
Topology and growth of a special class of holomorphic self-maps of $\mathbb{C}^{*}$
}

\author{
LINDA KEEN† \\ Department of Mathematics, Lehman College, Bronx, New York, 10468, USA
}

(Received 5 October 1987 and revised 2 June 1988)

Abstract. It is a general problem to find appropriate sets of moduli for families of functions that generate dynamical systems. In this paper we solve this problem for a specific family of holomorphic self-maps of $\mathbb{C}^{*}$ defined by

$$
\mathscr{F}=\left\{f \mid f=z^{n} \exp (P(z)+Q(1 / z))\right\} .
$$

The main theorem states that any function topologically conjugate to a member of $\mathscr{F}$ is holomorphically conjugate to some member of the family. It follows that the coefficients of the polynomials $P(z)$ and $Q(z)$ are a suitable set of moduli for the families of dynamical systems generated by these functions.

The moduli spaces of functions in $\mathscr{F}$ are easy to study computationally and have been studied by many authors. (See references in the text.)

\section{Introduction}

The motivation for the questions studied in this paper comes from the theory of complex analytic iteration. If two complex analytic maps are quasiconformally conjugate they have similar dynamical behavior. Given an analytic function $f$ therefore, a natural space to study is the moduli space of $f$; that is

$\theta(f)=\{g \mid g: \hat{\mathbb{C}} \rightarrow \hat{\mathbb{C}}$ is quasiconformally conjugate to $f\} /$ Möbius transformations.

To carry out this study one wants to find parameters for this space. Recently, this has been done for special families of functions. Among these are (see [BI, GGS, Mc]):

$\left\{f(z)=z^{2}+c ; c \in \mathbb{C}\right\}$, the 'quadratic family',

$\left\{f(z)=\lambda e^{z} ; \lambda \neq 0\right\}$, the 'exponential family' and

$\{f(z)=\sin (\alpha z+\beta) ; \alpha \neq 0\}$, the 'sine family'.

In this paper we consider the family of functions defined on the punctured plane $\mathbb{C}^{*}=\hat{\mathbb{C}}-\{0, \infty\}$ :

$$
\begin{gathered}
\mathscr{F}=\left\{f(z)=z^{n} \exp (P(z)+Q(1 / z)) ; n \in \mathbb{Z}, P \text { and } Q\right. \text { are } \\
\text { polynomials of degree } p \text { and } q, p+q>0\} .
\end{gathered}
$$

Our main theorem, Theorem 1, is that this family is 'topologically complete'; that is, THEOREM 1. Any holomorphic function on the twice punctured Riemann sphere which

$†$ Research supported in part by NSF grant \# DMS-8503015. 
is topologically conjugate to a member of the family $\mathscr{F}$ is holomorphically conjugate to a member.

It follows that the moduli space of such a function consists of members of the family and that the coefficients of the polynomials are parameters.

Any holomorphic self-map of the twice punctured Riemann sphere is holomorphically conjugate to one with essential singularities at 0 and $\infty$, so it has the form:

$$
g(z)=z^{m} \exp (E(z)+H(1 / z)),
$$

where $E$ and $H$ are entire functions.

Theorem 1 follows directly from

Proposition 3. If $\mathrm{g}$ is a holomorphic self-map of $\mathbb{C}^{*}$ and $\mathrm{g}$ is topologically conjugate to a function $f \in \mathscr{F}$, then $m=n$.

and

Theorem 2. If $\mathrm{g}$ is as in Proposition 3 and is conjugate to an element of $\mathscr{F}$ then $E$ and $H$ are polynomials.

In $\S 3$, we prove Proposition 3 by considering the change of argument along loops in $\mathbb{C}^{*}$. In $\$ 4$ we prove a lemma which says that $f$ and $g$ are "quasiconformally equivalent'; that is, there exist quasiconformal homeomorphisms $\psi_{1}$ and $\psi_{2}$ of the Riemann sphere such that $\psi_{1} \circ f=g \circ \psi_{2}$. In $\S 5$ we give estimates on the growth of the functions in $\mathscr{F}$, that together with the lemma, imply Theorem 2.

As an application of these ideas, in $\S 6$ we obtain analogous results for the family of entire functions:

$$
\tilde{F}=\left\{F(z)=n z+P\left(e^{z}\right)+Q\left(e^{-z}\right), P, Q \text { polynomials }\right\} .
$$

In $\S 7$ we discuss the special case of the functions

$$
f_{a, b}(z)=z \exp (z+a / z+b) .
$$

We describe some of the dynamical properties of these functions for various values of the parameters $a$ and $b$ and study their moduli spaces.

\section{Definitions and background material}

If a holomorphic self-map of $\mathbb{C}^{*}$ fails to be a covering of its image, the image point is called a singular value of the function. Critical values, the images of the critical points of a function, are singular values. They are assigned an order which is the order of branching, or the order of the zero of the derivative at the critical point. In addition to critical values, singular values can be asymptotic values or points in the closure of the set of critical and asymptotic values.

Definition 1. $w_{0}$ is an asymptotic value of the function $w=f(z)$ holomorphic on $\mathbb{C}^{*}$ if there is a path $\alpha:[0,1) \rightarrow \hat{\mathbb{C}}$ such that $\lim _{t \rightarrow 1} \alpha(t)=0$ or $\infty$ and $\lim _{t \rightarrow 1} f \circ \alpha(t)=w_{0}$. Definition 2. Let $w_{0} \in \hat{C}$ and let $U=\left\{w|0<| w-w_{0} \mid<\rho, \rho>0\right\}$. Then $w_{0}$ is a logarithmic singularity of the analytic function $w=f(z)$ if there exists a $\rho>0$ such that some connected component of $f^{-1} \mid U$ is simply connected.

Remark 1. It is not difficult to prove that isolated asymptotic values are logarithmic singularities (see [GK]). 
Proposition 1 (see [V]). An isolated essential singularity is an asymptotic value.

Proposition 2. The singular values of the functions in the family $\mathscr{F}$ are the asymptotic values 0 and $\infty$ and the critical values; that is, the images of the roots of the rational function

$$
z P^{\prime}(z)-(1 / z) Q^{\prime}(1 / z)+n=0 .
$$

Proof. Let $F(\eta)=n \eta+P\left(e^{\eta}\right)+Q\left(e^{-\eta}\right) . F(\eta)=\log \left(f\left(e^{\eta}\right)\right)$ for some choice of the logarithm. Finite asymptotic values of $F$ project onto finite (non-zero) asymptotic values of $f$ and finite (non-zero) asymptotic values of $f$ lift to finite asymptotic values of $F$. Since $F(\eta+2 \pi i)=F(\eta)+2 \pi n$, if $\alpha(t)$ is a path determining an asymptotic value $\omega$ for $F$ it stays in a horizontal strip and either $\operatorname{Re} \alpha(t) \rightarrow \infty$ or $\operatorname{Re} \alpha(t) \rightarrow-\infty$ while $|\operatorname{Im} \alpha(t)|$ stays bounded. If $n \neq 0$ it is clear that $\omega=\infty$. If $n=0$, $F$ is rational in $e^{\eta}, f$ is the exponential of a rational map whose only poles are at 0 and $\infty$, and its only asymptotic values are 0 and $\infty$.

\section{Proof of Proposition 3}

Proposition 3. If $f$ and $g$ are holomorphic self-maps of $\mathbb{C}^{*}$ with essential singularities at 0 and /or $\infty$ given by

$$
\begin{aligned}
& f(z)=z^{n} \exp (P(z)+Q(1 / z)), P, Q \text { entire, } n \in Z, \\
& g(z)=z^{m} \exp (E(z)+H(1 / z)), E, H \text { entire, } m \in Z,
\end{aligned}
$$

and $f$ and $g$ are topologically conjugate then $m=n$.

Proof. Suppose that $f$ and $g$ are topologically conjugate: that is,

$$
\varphi \circ g=f \circ \varphi, \quad \psi=\varphi^{-1}
$$

where $\varphi, \psi$ are homeomorphisms of $\mathbb{C}^{*} \rightarrow \mathbb{C}^{*} . \varphi$ and $\psi$ can be continuously extended to map $\{0, \infty\}$ to $\{0, \infty\}$; the points may either be left fixed or interchanged.

Let $\gamma$ be the closed path $\gamma:[0,1] \rightarrow \mathbb{C}^{*}, \gamma(t)=\exp (2 \pi \mathrm{i} t) ; \gamma$ separates 0 and $\infty$. Its image, $\varphi \gamma$, also separates 0 and $\infty$ and as $z$ traverses $\gamma$, its image, $w=\varphi(z)$ traverses $\varphi \gamma$ and $\arg w$ changes by $2 \pi \varepsilon, \varepsilon= \pm 1$. We claim now that $\arg f(w)=$ $\arg f \circ \varphi(z)$ increases by $2 \pi n \varepsilon$ as $z$ traverses $\gamma$. This follows since it is true for $\arg \varphi^{n}(z)$ and the image of $\varphi \gamma$ under $z \rightarrow P(z)+Q(1 / z)$ is a well defined closed curve in the plane; the exponential maps this closed curve onto one which has zero net change in its argument. Finally, the change in $\arg \psi \circ f \circ \varphi$ is $\varepsilon \cdot 2 \pi \varepsilon=2 \pi n$ as $z$ traverses $\gamma$.

A similar argument shows that the change in the argument of $g$ along $\gamma$ is $2 \pi m$; hence $m=n$.

\section{The main technical lemma}

If $\varphi$ is a homeomorphism of the sphere which conjugates a function $f$ to a function $g$, then $\varphi$ takes the singular set of $f$ to that of $g$, taking critical values to critical values of the same order and logarithmic singularities to logarithmic singularities. It also takes critical points to critical points. It follows that if $f \in \mathscr{F}$, then $g$ has finitely many critical points and values, and has 2 logarithmic singularities. Since we are only concerned about conjugacy up to Möbius transformations we can assume that $\varphi$ fixes 0 and $\infty$. 
It is generally thought that functions which are topologically conjugate are quasiconformally conjugate but except in a few special cases [Sul] this has not been proved. Here we prove a weaker statement.

MAIN Lemma. Let $f \in \mathscr{F}$ be given and let $g$ be topologically conjugate to it by $\varphi$ as above. Then there exist quasiconformal homeomorphisms of the sphere fixing 0 and $\infty, \psi_{1}$ and $\psi_{2}$, such that $\psi_{2} \circ f=g \circ \psi_{1}$.

Remark 2. Künzi, Wittich and Pöschl used quasiconformal mappings in their work on value distribution theory (see [Ku, Wi2, Po]). The idea of the proof below grew out of reading their work. They did not have the techniques we use at their disposal; these make the constructions below much simpler than theirs. Moreover, the proof below can easily be adapted to prove the theorems in their papers.

Proof. Let $S=\left\{\beta, a_{1}, \ldots, a_{k}\right\}$ where the $a_{i}, i=1, \ldots, k$ are the critical values of $f$ and $\beta$ is a point in $\mathbb{C}^{*}$ distinct from the critical points and critical values of $f$. We can find an isotopy $\Phi: \hat{C} \times[0,1] \rightarrow \hat{\mathbb{C}}$ such that:

$$
\begin{aligned}
& \Phi(z, 0)=\varphi(z), \\
& \Phi(p, t)=\varphi(p) \quad p \in S,
\end{aligned}
$$

and $\Phi(z, 1)=\psi_{2}(z)$ is quasiconformal (see Proposition 2, [Be2]).

The maps $f: \mathbb{C}^{*}-f^{-1}(S) \rightarrow \mathbb{C}^{*}-S$ and $g: \mathbb{C}^{*}-g^{-1} \circ \varphi(S) \rightarrow \mathbb{C}^{*}-\varphi(S)$ are covering maps. Therefore, by the homotopy lifting theorem, $\Phi(t, z)$ lifts to a homotopy $\tilde{\Phi}(t, z)$ such that

$$
\begin{gathered}
\tilde{\Phi}(z, 0)=\varphi(z), \\
g \circ \tilde{\Phi}(z, t)=\Phi(f(z), t)
\end{gathered}
$$

and $\tilde{\Phi}(z, t)$ are homeomorphisms.

Now for any $\beta^{\prime} \in f^{-1}(\beta), g \circ \tilde{\Phi}\left(\beta^{\prime}, t\right)=\Phi\left(\beta^{\prime}, t\right)=\varphi(\beta)$. Since $\Phi$, is continuous and $g^{-1} \circ \varphi(\beta)$ is discrete, $\tilde{\Phi}\left(\beta^{\prime}, t\right)$ is constant. Therefore if $\beta_{n}$ is a sequence which tends to $\infty$ and $\gamma_{n}$ is a sequence which tends to 0 , we can extend the isotopies $\tilde{\Phi}$ and $\Phi$ to $\hat{\mathbb{C}}$ so that they fix 0 and $\infty$ for all $t$.

Let $\omega_{1}=\tilde{\Phi}(z, 1)$. Then since $f$ and $g$ are analytic and $\psi_{2}$ is quasiconformal, by the functional relation

$$
g \circ \psi_{1}=\psi_{2} \circ f
$$

and the removable singularity theorem, $\psi_{1}$ is also quasiconformal; moreover, by the above, $\psi_{1}$ and $\psi_{2}$ fix 0 and $\infty$.

\section{Proof of the main theorem}

We are now ready to prove

Theorem 2. If $g=z^{n} \exp (E(z)+H(1 / z)), E$ and $H$ entire, and if $g$ is topologically conjugate to a function $f \in \mathscr{F}, f=z^{n} \exp (P(z)+Q(1 / z)), P$ and $Q$ polynomials of degrees $p$ and $q$ respectively, then $E$ and $H$ are also polynomials of degrees $p$ and $q$ respectively.

Proof. By the Hölder continuity of any quasiconformal map, there exists $K>0$ such 
that:

$$
\begin{aligned}
& |z|^{1 / K} \leq\left|\psi_{2}(z)\right| \leq|z|^{K}, \\
& |z|^{1 / K} \leq\left|\psi_{1}(z)\right| \leq|z|^{K}
\end{aligned}
$$

By hypothesis:

$$
f(z)=z^{m} \exp (P(z)+Q(1 / z))
$$

where

$$
P(z) \text { is a polynomial of degree } p
$$

and

$$
Q(z) \text { is a polynomial of degree } q .
$$

As $|z|=r \rightarrow \infty,(5.1)$ and (5.2) imply

$$
\begin{aligned}
M\left(r, \psi_{2} \circ f \circ \psi_{1}^{-1}\right) & \leq \underset{|z|=r}{\operatorname{Max}}\left|\psi_{2}\left\{\exp \left(\operatorname{Re}\left(P \circ \psi_{2}^{-1}\right)+0(1)\right)\right\}\right| \\
& \leq\left\{\exp \left(c\left(r^{K}\right)^{p}\right\}^{K}, \quad \text { for some } c>0\right. \\
& =\exp \left(c^{\prime} e^{K^{2} p}\right),
\end{aligned}
$$

where $M(r, g)$ is the maximum modulus on $|z|=r$.

But $M\left(r, \psi_{2} \circ f \circ \psi_{1}^{-1}\right)=M(r, g)$ where

$$
g(z)=z^{m} \exp (E(z)+H(1 / z)), \quad E, H \text { entire, }
$$

so it follows from (5.3) that as $|z|=r \rightarrow \infty$, we have

$$
\operatorname{Re}\left(E(z)=O\left(r^{K^{2} p}\right), H(1 / z) \rightarrow\right. \text { constant; }
$$

hence $E$ is a polynomial.

Similarly, by considering the behavior of $f$ and $g$ as $|z| \rightarrow 0$ we see that $H$ is also a polynomial, and $g \in \mathscr{F}$.

We now show that the degree of $E$ is $p$ and the degree of $H$ is $q$. Consider the asymptotic behavior of $f$ at $\infty: w=P(z)+Q(1 / z)$ is analytically conjugate to $z \rightarrow z^{p}$ in a neighborhood of $\infty$ so is a $p$-fold covering of a punctured disk at $\infty$; composing with the exponential we have that $f$ is asymptotically $\exp \left(z^{p}\right)$ (this grows faster than any polynomial so the $z^{n}$ factor can be ignored). Since $\psi_{1}$ and $\psi_{2}$ are quasiconformal, fix $\infty$ and satisfy (5.1), $\psi_{1} \circ f$ has the same asymptotic behavior. Therefore so do $g \circ \psi_{2}$ and $g$. It follows that $E(z)$ must also be a $p$-fold covering of a punctured disk at $\infty$ and have degree $p$. The same argument applied to $Q$ and $H$ show that they have the same degrees. Proposition 3 and Theorem 2 immediately imply

THEOREM 1. If $g$ is a holomorphic self-map of $\mathbb{C}^{*}$ and $g$ is topologically conjugate to a function $f \in \mathscr{F}$, then $g$ is holomorphically conjugate to some $h \in \mathscr{F}$.

As a corollary we have:

COROLlaRY 1. The map to the coefficients gives a faithful representation of $\theta(f)$ to a subset of $\mathscr{F}$ and so the dimension of the space is at most $p+q$.

Proof. An element of $\theta(f)$ is an equivalence class of functions; in each class we can choose a representative $g$ whose essential singularities are at 0 and $\infty$. This representative is defined up to conjugation by the stretch map $z \rightarrow a z$ where $a$ is 
chosen so that

$$
g(z)=z^{n} \exp \{E(z)+H(1 / z)\}
$$

and $E$ is a monic polynomial of degree $p$ and $H$ is a polynomial of degree $q$. $E$ and $H$ therefore have $p+q$ coefficients since the constant terms combine. This map to the coefficients gives a faithful representation $\theta(f) \rightarrow \mathscr{F}$.

In [Kn], we prove that the dimension of $\theta(f)$ is at most $p+q$ by counting singular values, normalizations and conjugating parameters.

\section{An application}

Consider the family of entire transcendental functions

$$
\begin{aligned}
\tilde{\mathscr{F}}=\{ & F(\eta)=n \eta+P\left(e^{\eta}\right)+Q\left(e^{-\eta}\right), P \text { and } Q \text { polynomials } \\
& \text { of degrees } p \text { and } q \text { respectively }\} .
\end{aligned}
$$

Functions in this family commute with the group of translations $\Gamma$ of the plane generated by

$$
T: \xi \rightarrow \xi+2 \pi \mathrm{i}
$$

That is,

$$
F(\eta+2 \pi \mathrm{i})=F(\eta)+2 \pi n \mathrm{i}
$$

There is an obvious isomorphism between the family $\tilde{F}$ modulo this translation group and the family $\mathscr{F} ; \alpha:[F] \leftrightarrow f$ given by $f=\exp \circ F \circ \log$.

If $f_{1}$ and $f_{2}$ are conjugate by a stretch map $z \rightarrow a z$ then $\alpha^{-1}\left(f_{1}\right)$ and $\alpha^{-1}\left(f_{2}\right)$ are conjugate by a translation $\eta \rightarrow \eta+b$ where $b$ is defined modulo $2 \pi$ i.

In general, for $F \in \mathscr{F}, \theta(F) \subset \mathscr{F}$, and $\theta(F)$ is infinite dimensional; for example, if $F$ possesses a wandering domain whose iterates are identified by elements of $\Gamma$, $\theta(F)$ contains quasiconformal deformations of $F$ (see $\S 7$ ) which do not commute with $\Gamma$. The subspace of $\tilde{F}$ consisting of functions which commute with $\Gamma$ is finite dimensional and isomorphic to $\theta(f), f=\alpha([F])$. These results follow from those of $[\mathrm{Kn}]$. However, the results of this paper allow us to say more.

COROLlaRY 2. The subspace of $\theta(F)$ consisting of functions which commute with the translation group is precisely the subspace of $\tilde{\mathscr{F}}$ in which $P$ is monic and the coefficients of the polynomials are moduli.

Proof. This follows directly from the isomorphism between $\mathscr{F}$ and $\mathscr{F}$ and the proof of Corollary 1. If $\varphi: \mathbb{C} \rightarrow \mathbb{C}$ is a quasiconformal map such that $G=\varphi \circ F \circ \varphi^{-1}$ is entire and commutes with $\Gamma$ then $G^{\prime}$ is entire and periodic with period $2 \pi \mathrm{i}$. It follows that $G(z)=H\left(e^{z}\right)+n z$ where $H$ is meromorphic in $e^{z}$. Set

$$
g=\exp \circ G \circ \log \text {. }
$$

Then,

$$
\begin{aligned}
g & =\exp \circ \varphi \circ F \circ \varphi^{-1} \circ \log \\
& =\exp \circ \varphi \circ \log \circ f \circ \exp \circ \varphi^{-1} \circ \log .
\end{aligned}
$$

Now,

$$
\psi=\exp \circ \varphi \circ \log
$$

is quasiconformal so $g \in \theta(f)$. 


\section{An example}

In [Kn], we studied the dynamics of holomorphic self-maps of $\mathbb{C}^{*}$, and considered the special family:

$$
f_{\Lambda}(z)=z \exp (z+1 / z+\lambda)
$$

for some complex values of $\lambda$. Computational experiments showed that as $\lambda$ varied in the complex plane there were many 'Mandelbrot sets'. For $\lambda$ in these sets, $f_{\lambda}$ has some stable behavior. The experiments also indicated that the set of $\lambda$ 's for which the Julia set is the whole sphere has positive measure.

Let us now consider the family

$$
\mathscr{F}=\left\{f_{b, c}(z)=z \exp (z+b / z+c) ; b \in \mathbb{C}^{*}, c \in \mathbb{C}\right\} .
$$

Set $b=1$ and $c=-2.5$. It is not too hard to check that $f_{1,-2.5}$ has two fixed points on the positive axis, one attracting and one repelling. There is a critical point on the positive axis which is attracted to the attracting fixed point. The other critical point is on the negative real axis; it is in the Julia set. The stable set is the basin of attraction of the attracting fixed point. The function commutes with complex conjugation since $b$ and $c$ are real.

As an application of the main theorem we can prove:

Proposition 4. $\theta\left(f_{1,-2.5}\right)$ parametrized by $b$ and contains the real subvariety:

$$
V=\left\{b>0, c \text { real, } 4 b<c^{2}<4 b+4\right\} \text {. }
$$

Proof. It is straightforward to check that there are, in general, two fixed points for any $f_{b, c} \in \mathscr{F}$ and that at most one of them can be attracting. One is attracting if $\left|1-\sqrt{ }\left(c^{2}-4 b\right)\right|<1$. If $c^{2}=4 b$, there is only one fixed point and it is indifferent. If $b$ and $c$ are real, $b>0$, one critical point is attracted to the fixed point and the other is in the Julia set. Therefore, if a function is in $V$, its critical orbits behave like those of $f_{1,-2.5}$.

A function in $V$ has a forward invariant stable component $D$ containing the attracting fixed point and the critical value. Remove the orbit of the fixed point from $D$ and call the result $\hat{D}$. Identify points of $\hat{D}$ by the grand orbit relation; that is, $x \approx y$ if there are integers $m$ and $n$ such that $f^{n}(x)=f^{m}(y)$. $\hat{D}$ modulo this relation is a torus. Puncture this torus at the grand orbit of the critical value. Call the resulting punctured torus $T_{b, c}$.

Let $\varphi$ be any quasiconformal homeomorphism of $T_{b, c}$ onto $T_{1,-2.5}$ and let $\tilde{\mu}$ be its Beltrami differential. Lift this differential to $\hat{D}$ and obtain a measurable structure $\mu$ there. Spread this structure to the preimages of $\hat{D}$ using the function $f$; that is,

$$
\mu\left(f^{n}(z)\right) \overline{f^{n \prime}(z)} / f^{n \prime}(z)=\mu(z), \quad z \in f^{-n}(\hat{D}) .
$$

Define $\mu \equiv 0$ on the complement of the stable set. Since there can be only one stable orbit by the classification theorem [Sul], $\mu$ is defined everywhere except on the grand orbit of the attractive fixed point which is a set of measure zero. Now the measurable Riemann mapping theorem implies there is a $\mu$-quasiconformal mapping conjugating $f_{b, c}$ to $f_{1,-2.5}$.

Remark 3. Since $f_{1,-2.5}$ leaves the positive and negative axes invariant, each function in $\theta\left(f_{1,-2.5}\right)$ also has invariant curves joining its essential singularities. 
Remark 4. If $c^{2} / 4-1<b<0, f_{b, c}$ has an attractive fixed point which attracts both critical values. These functions are clearly not in any class in $\theta\left(f_{1,-2.5}\right)$.

Remark 5. The punctured tori obtained from the functions in $V$ are all rectangles or rhombi; that is, if $\tau$ is their classical modulus (in the upper half plane), it has the form $\tau=\mathrm{it}, t>0$ or $|\tau|=1$. This is because for $b$ and $c$ real, $f_{b, c}$ commutes with complex conjugation. This involution descends to the torus $T_{b, c}$ and the rectangles and rhombi are the only tori which admit involutions.

Remark 6. The computer experiments mentioned above, for $b=1, c$ complex, show that period doubling takes place on the line $b=1$, below the curve $c^{2}=4 b+4$. This evidence, together with the fact that $f_{b, c}$ with non-symmetric tori must be accounted for, make it clear that $V$ can be 'thickened' to include 2 complex-dimensional Mandelbrot type sets. It would be interesting to describe explicitly the break-up of the $(b, c)$ parameter space into its various moduli spaces.

\section{Acknowledgement}

The author would like to extend her thanks to I. N. Baker who read earlier versions of this paper and provided substantial simplifications of the proofs of the main theorems.

\section{REFERENCES}

[A] L. V. Ahlfors. Lectures on Quasiconformal Mappings. Van Nostrand, New York, 1966.

[AB] L. V. Ahlfors and L. Bers. Riemann's mapping theorem for variable metrics. Ann. of Math. 72 (1960).

[Be1] L. Bers. Quasiconformal mappings and Teichmüller's theorem, in Analytic Functions. Princeton, 1960, pp. 89-119.

[Be2] L. Bers. Uniformization by Beltrami equations. Comm. Pure and Applied Math. 143 (1961), pp. 215-228.

[BI] P. Blanchard. Complex analytic dynamics on the Riemann Sphere. Bull. A.M.S. 11 (1984).

[GGS] E. Ghys, L. Goldberg and D. Sullivan. On the measurable dynamics of $z \rightarrow \exp (z)$. Ergod. Th. \& Dynam. Sys. 5 (1985).

[GK] L. Goldberg \& L. Keen. A finiteness theorem for a dynamical class of entire functions. Ergod. Th. \& Dynam. Sys. 6 (1986), 183.

[Kn] L. Keen. Dynamics of holomorphic maps of $\mathbb{C}^{*}$, in Holomorphic Functions and Moduli. Vol. I, ed. D. Drasin, Springer, Berlin-New York, 1988.

[Ku] H. Künzi. Quasikonforme Abbildungen, Springer-Verlag, Berlin-New York, 1960.

[LV] O. Lehto \& K. Virtanen. Quasiconformal Mappings in the Plane, Springer-Verlag, Berlin-New York, 1973.

[LeV] Thiem, Le-Van. Über das Umkehrproblem der Wertverteilungslehre. Comment. math. Helv. 23 (1949).

[McM] C. McMullen. Area and Hausdorff dimension of Julia sets of entire functions. Trans. AMS 300 (1987), 1, 329-342.

[N] R. Nevanlinna. Analytic Functions. Springer-Verlag, Berlin-New York, 1970.

[Po] K. Pöschl. Über die Wertverteilung der erzeugenden Funktionen Riemannscher Flächen mit endlich vielen periodischen Enden. Math. Ann. 123 (1951), Helv. 2 (1930).

[Sul] D. Sullivan. Quasiconformal homeomorphisms and dynamics III, Acta Math. to appear.

[Tei] O. Teichmüller. Untersuchungen über konforme und quasikonforme Abbildungen. Deutsche Math. 3 (1938).

[V] G. Valiron. Theory of Integral Functions. Chelsea, 1949.

[Wi1] H. Wittich. Zum Beweis eines Satzes uber quasikonforme Abbildungen. Math. Zeit. 51 (1948).

[Wi2] H. Wittich. Über die Wachstumsordung einer ganzen transzendenten Funktion. Math. Zeit. 51 (1948). 